\title{
Hemophilia gene therapy comes of age
}

\author{
Lindsey A. George \\ Division of Pediatrics, The Children's Hospital of Philadelphia, Philadelphia, PA; and Department of Pediatrics, Perelman School of Medicine at the University of Pennsylvania, \\ Philadelphia, PA
}

Concurrent with the development of recombinant factor replacement products, the characterization of the $F 9$ and $F 8$ genes over 3 decades ago allowed for the development of recombinant factor products and made the hemophilias a target disease for gene transfer. The progress of hemophilia gene therapy has been announced in 3 American Society of Hematology scientific plenary sessions, including the first "cure" in a large animal model of hemophilia B in 1998, first in human sustained vector-derived factor IX activity in 2011, and our clinical trial results reporting sustained vector-derived factor IX activity well into the mild or normal range in 2016. This progression to clinically meaningful success combined with numerous ongoing recombinant adeno-associated virus (rAAV)-mediated hemophilia gene transfer clinical trials suggest that the goal of gene therapy to alter the paradigm of hemophilia care may soon be realized. Although several novel therapeutics have recently emerged for hemophilia, gene therapy is unique in its potential for a one-time disease-altering, or even curative, treatment. This review will focus on the prior progress and current clinical trial investigation of rAAV-mediated gene transfer for hemophilia A and B.

\section{Introduction}

Five years after the first successful continuous expression of clotting factor after gene transfer ${ }^{1,2}$ and 2 decades into clinical trial investigation, gene therapy is poised to become a therapeutic alternative to clotting factor concentrate for hemophilia patients. Bleeding manifestations of hemophilia are highly predictable based on circulating factor activity, wherein the vast majority of bleeding sequelae and spontaneous hemorrhage occur in patients with severe (factor activity $<1 \%$ of normal) or moderate (factor activity $1 \%$ to $5 \%$ of normal) hemophilia. ${ }^{3}$ Although there is not yet a conclusive minimal desired target for transgene-derived factor activity, recently reported postgene transfer factor VIII (FVIII) and factor IX (FIX) activity well into the mild or normal range is anticipated to confer a large clinical impact. Further, although preliminary clinical data as summarized in this review are promising, long-term safety and efficacy will only become available with mature clinical experience. This review will provide a summary of prior and current clinical progress and challenges of recombinant adeno-associated virus (rAAV)-mediated gene transfer for hemophilia $A(H A)$ and hemophilia $B(H B)$.

\section{Adeno-associated virus vectors}

Although several different gene delivery vehicles, or vectors, have been evaluated in hemophilia gene transfer clinical trials, current efforts have coalesced around the use of rAAV vectors for in vivo hepatocyte gene delivery. ${ }^{4}$ Additional strategies are in preclinical investigation and reviewed elsewhere. ${ }^{5}$ Naturally occurring adeno-associated virus (AAV) is a member of the Parvoviridae family; it is generally regarded as nonpathogenic and is replication defective. rAAV vectors are engineered from AAV by deleting the AAV coding sequences

Submitted 29 June 2017; accepted 22 August 2017. DOI 10.1182/ bloodadvances. 2017009878 .
This article was selected by the Blood Advances and Hematology 2017 American Society of Hematology Education Program editors for concurrent submission to Blood Advances and Hematology 2017. It is reprinted in Hematology Am Soc Hematol Educ Program. 2017;2017:587-594.

(C) 2017 by The American Society of Hematology 
and replacing these with the gene of interest under the control of an appropriate promoter. The recombinant virion thus consists of a nucleic acid sequence (transgene) and an ordered set of proteins (capsid) responsible for guiding the transgene to the target cell. From a safety standpoint, AAV requires a helper virus (eg, adenovirus) for replication and is, therefore, replication defective and nonpathogenic in vivo. Furthermore, rAAV-mediated gene transfer results in an episomally retained transgene and is predominantly nonintegrating. rAAV vectors, therefore, carry minimal risk of insertional mutagenesis. ${ }^{4,6}$ In terms of efficacy, rAAV is able to transduce postmitotic cells (eg, hepatocytes) and has shown long-term expression in small and large immunocompetent $\mathrm{HA}$ and $\mathrm{HB}$ animal models and humans. ${ }^{1,2,7-9}$

For purposes of designing a vector cassette, the AAV genome is $4.7 \mathrm{~kb}$ and consists of inverted terminal repeats and $145 \mathrm{bp}$. The inverted terminal repeats must be retained as bookends of the transgene to allow for AAV vector packaging. Of relevance for hemophilia gene therapy, rAAV is able to package the complementary DNA of FIX (1.5 kb) and single-stranded B-domain-deleted FVIII (BDD-FVIII; 4.4 kb). ${ }^{10}$ Vector production occurs in cell culture, which provides the basic biosynthetic machinery, but the presence of additional genes is required to direct vector generation and packaging of the desired transgene in the vector virion. These additional genes include AAV rep and cap as well as adenovirus helper genes to support replication of the vector genome. ${ }^{11}$

Although all current hemophilia gene therapy trials use rAAV vectors with the goal to achieve translation of 1 of 3 proteins (wildtype FIX, FIX-Padua, or B domain-deleted FVIII), the vectors can vary significantly in terms of manufacturing procedures and composition. Current rAAV vectors in hemophilia clinical investigation are produced either in a mammalian expression system (most commonly, HEK293 cells) or by transfection of insect cells (Spodoptera frugiperda) with baculovirus. ${ }^{12}$ The use of insect cell lines is attractive for their high-titer vector production capacity. However, preliminary work has shown that there are extensive differences in capsid posttranslational modifications in insect vs mammalian vector production systems. ${ }^{13}$ Whether these posttranslational differences influence vector immunogenicity and/or expression kinetics is not currently known. All rAAV manufacturing procedures result in concurrent production of empty capsids or "empties" (fully assembled capsids devoid of transgene DNA) but differ in their ability to isolate and remove empty capsids. The role of empty capsids in vector safety and efficacy and the significance of their variability within clinical trials are unclear. In addition to differences in manufacturing platforms and procedures, vectors vary in their cassette genome, including the transgene itself or configuration (self-complementary or single stranded), promotor, enhancer, codon optimization, and content of cytosine guanine dinucleotide motifs. Differences in genome configuration may alter transduction and expression efficiency and in the case of cytosine guanine dinucleotide motifs, by serving as a ligand for Toll-like receptor 9 , contribute to eliciting an innate immune response that may further stimulate an adaptive immune response. ${ }^{14}$ Lastly, since the initiation of rAAV gene transfer trials for hemophilia, several naturally occurring AAV serotypes have been identified and can be distinguished by their tissue specific tropism. ${ }^{15}$ Current hemophilia clinical trials include the use of naturally occurring (AAV5, AAV6, AAV8) and bioengineered (AAV-rh10, AAV-Spark100, AAV-Spark200) AAV serotypes.

Work over the past 5 years has provided evidence that rAAV vectors are approaching their promise to turn gene transfer for hemophilia into a therapeutic reality. This has occurred in the broader context of successful licensing of 2 gene therapy products in Europe, making it clear that a regulatory pathway exists for genebased products. ${ }^{16,17}$ The feasibility of bringing a gene-based therapy through to licensure coupled with promising results for hemophilia gene therapy in clinical trials have translated into support for clinical development by a range of large and small pharmaceutical and biotech companies. This has provided better resources to conduct clinical trials in this area compared with earlier efforts that were academic based. As a result, nearly all current hemophilia gene transfer trials are industry sponsored. Although this industry commitment allows for resources to pursue a more rapid pace of progress, it necessitates that the details of individual vector manufacturing procedures and genome configuration are largely proprietary and thus, are not disclosed. The contribution of undisclosed variability between vectors is of unclear clinical significance but may be relevant for understanding variation in clinical trial results.

Approximately 40 rAAV gene transfer protocols have been approved by the Food and Drug Administration, ${ }^{18}$ and over the preceding 15 years, $\sim 65$ hemophilia patients have undergone in vivo liver-directed gene transfer with rAAV vectors. ${ }^{1,2,19-24}$ Although appropriate continued caution exists regarding long-term or late adverse events, no major safety concerns have been identified in clinical investigation to date. The short-term risks associated with rAAV, at this point, are well delineated and namely limited to an anticapsid immune response. Although this immune response has not posed a major safety concern, it threatens to limit efficacy and is discussed in detail in this review.

The definitive assessment of long-term risks of rAAV hepatocyte transduction will require mature clinical data, but extensive preclinical studies have addressed the theoretical risk of genotoxicity. Because rAAV is predominantly nonintegrating, it carries minimal risk of insertional mutagenesis. However, direct sequencing of DNA from target organs in animals has shown that integration can occur. ${ }^{25,26}$ Furthermore, a single preclinical murine study documented hepatocellular carcinoma (HCC) after liver-directed in vivo rAAV gene delivery in neonatal mice. ${ }^{27}$ A subsequent comprehensive study showed that rAAV vector dose, promotor, enhancer, and the degree of cell division during vector administration all affected HCC risk in murine studies of rAAV gene delivery. This work supported using the lowest possible vector dose, targeting postmitotic hepatocytes, and using a liver-restricted promoter, such as human $\alpha$-1-antitrypsin, to minimize the theoretical risk of insertional mutagenesis previously identified in preclinical studies. ${ }^{28}$

More recent analysis of human HCC samples showed clonal integration of wild-type AAV2 at known proto-oncogenes, raising concern for an association of wild-type AAV2 with oncogenic insertional mutagenesis in human HCC. ${ }^{29}$ However, the clonal integration was observed in $7 \%$ of $\mathrm{HCC}$ tissues and $22 \%$ of adjacent healthy liver tissues, consistent with prior work showing that wild-type AAV2 has antioncogenic properties. ${ }^{29,30}$ Thus far, there have been no genotoxic events observed in preclinical large animal models or human clinical trials of liver-directed rAAVmediated gene transfer. This includes 8-year follow-up data from canine HB dog models, ${ }^{31}$ 3-year follow up of $10 \mathrm{HB}$ subjects with sustained FIX expression, ${ }^{2}$ and 14-year follow up of 4 subjects with nonsustained transgene expression (Lindsey A. George and Katherine A. High, unpublished data). 


\section{Lessons learned from early in vivo rAAV liver-directed FIX trials: AAV2-FIX and AAV8-FIX}

Although well-characterized murine and canine hemophilia models allowed for extensive disease-specific investigation, preclinical models failed to fully predict or subsequently recapitulate the human immune response to $\mathrm{rAAV} .^{8,32}$ As a result, our understanding of the immune response to $\mathrm{rAAV}$ is limited to clinical trial investigation. The first human $\mathrm{AAV}$ liver-directed gene therapy trial for hemophilia used an AAV2-FIX vector. ${ }^{33}$ Although only 7 subjects were infused with vector, the trial identified the 2 major obstacles for sustained transgene expression of rAAV gene transfer: limitations posed by the human humoral and cellular immune response to rAAV.

\section{Humoral immune response: AAV neutralizing antibodies}

Preexisting antibodies to AAV (neutralizing antibodies [NAbs]) are present in $\sim 30 \%$ to $50 \%$ of the population. ${ }^{34}$ Although AAV NAbs do not block vector transduction when the vector is delivered directly into the target tissue, ${ }^{33}$ they do inhibit target cell transduction when $\mathrm{rAAV}$ vector is delivered via the circulation. ${ }^{19}$ This was first noted in the AAV2-FIX liver trial and is shown by the peak factor IX activity (FIX:C) of subjects $E$ and $F$ who were treated at the same dose cohort of $2 \times 10^{12}$ vector genomes $(\mathrm{vg}) / \mathrm{kg}$. Subject $E$, who had a low-titer preexisting AAV NAb (1:2), achieved peak transgene FIX:C of $\sim 10 \%$, whereas subject $\mathrm{F}$, who had an NAb titer of 1:17, did not show appreciable FIX expression. ${ }^{19}$ As a result, subsequent trials restrict enrollment to subjects with no- or low-titer preexisting AAV NAb. One potential source of variability among results from different sponsors is the sensitivity of the assay used to screen for these antibodies. If low-titer antibodies are missed by a less sensitive assay, datasets will contain subjects who fail to exhibit any appreciable transgene expression and/or result in a wide range of transgene expression at the same vector dose.

\section{Cellular immune response to the AAV capsid}

The AAV2 liver trial additionally observed a cellular immune response to the rAAV capsid. This was first observed in subject $E$, who received a vector dose of $2 \times 10^{12} \mathrm{vg} / \mathrm{kg}$. Subject $E$ reached peak transgene-derived FIX:C of $\sim 10 \%$ that declined accompanied by a rise in alanine aminotransferase (ALT) and aspartate aminotransferase. Interferon- $\gamma$ Enzyme-Linked ImmunoSpot and subsequently pentamer data from the next subject followed a similar course, suggesting clonal expansion of rAAV capsid-specific CD8 + T cells. This work supported the hypothesis that loss of transgene expression was caused by an AAV capsidspecific CD8+ T-cell response to capsid peptides presented via major histocompatibility complex class I on the surface of transduced hepatocytes, resulting in a cytolytic effect on the transduced cells and loss of expression (Figure 1). ${ }^{35,36}$ This is detected clinically by transaminase elevation and a decline in FIX:C. To date, clearance of transduced hepatocytes in the setting of an immune response in hemophilia rAAV gene transfer trials has not posed clinical concern for hepatic damage. Although it has not posed major safety concerns, it leads to loss of transgene expression and thereby, limits efficacy. Importantly, interpretation of trial subject transaminase values requires careful attention and stringent follow

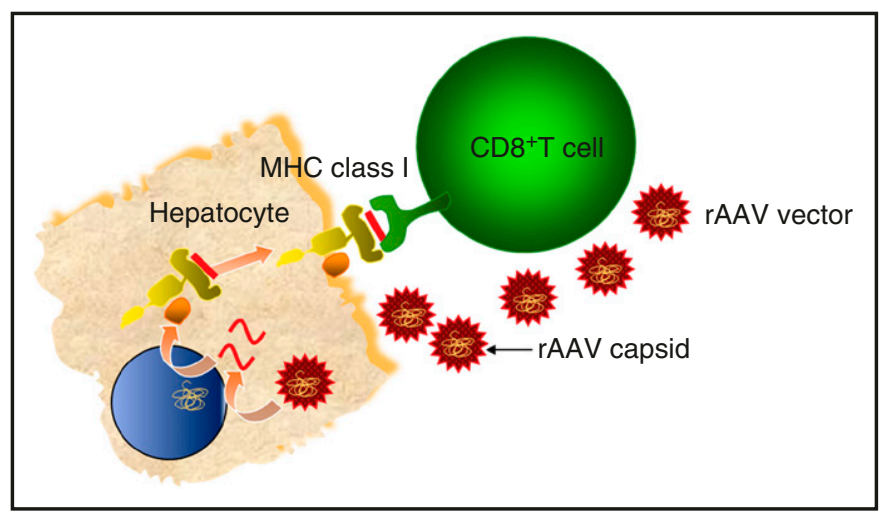

Figure 1. Hypothesis of the cellular immune response to hepatocytes after rAAV transduction. rAAV enters the hepatocyte by receptor-mediated endocytosis, wherein the vector genome, including the transgene, is released from the capsid (uncoating). Thereafter, the capsid is degraded in the cytosol, and capsid peptides are loaded onto major histocompatibility complex (MHC) class I on the surface of the transduced hepatocytes. Presentation of capsid peptides on MHC class I triggers a cellular immune response to the transduced hepatocytes, resulting in clearance of the hepatocyte.

up. Although evidence of an immune response may be represented by transaminases that rise above normal values, frequently values only rise above the individual subject baseline measurement while remaining within normal limits. ${ }^{20-23}$ That said, evaluation of transaminases for evidence of an immune response can be confounded by routine variation, such as preanalytical hemolysis or subject alcohol consumption, making the interpretation of subtle variation in transaminase values complicated.

The next generation rAAV HB trial used an AAV8 vector and incorporated observations from the AAV2-FIX trial by including a provision to use steroids in the setting of a possible rAAV capsid immune response. In a seminal study, Nathwani et al achieved the first successful vector-derived sustained FIX:C of $2.9 \%$ to $7.2 \%$ of normal in the highest-dose cohort $\left(2 \times 10^{12} \mathrm{vg} / \mathrm{kg}\right){ }^{1,2}$ When the investigators noted a rise in ALT and decrease in FIX:C, they initiated a course of prednisone and thereafter observed a decline in ALT and sustained transgene expression. Four of 6 patients treated in the high-dose therapeutic cohort $\left(2 \times 10^{12} \mathrm{vg} / \mathrm{kg}\right)$ required a 4- to 12-week course of steroids to control an AAV capsid-immune response and maintain transgene expression. Although all subjects had sustained FIX:C, 3 of 4 patients who required steroids lost $50 \%$ to $70 \%$ of peak transgene expression. Thus, in most cases, steroids were able to rescue some but not all FIX expression, supporting that the requirement for steroids should be minimized.

The investigators also noted that minimizing the time from first noted rise in ALT to initiation of steroids was important for maintaining maximal transgene expression. Specifically, waiting 2 vs 7 days to initiate steroids from time of initial ALT rise resulted in $0 \%$ and $70 \%$ reduction in peak transgene activity, respectively. This highlighted the importance of vigilant laboratory surveillance and timely intervention with steroids by the investigator. Although the precise timing of the capsid immune response varies among vectors, this immune response in AAV hemophilia gene transfer trials, to date, has occurred within the first 12 weeks after vector infusion. ${ }^{1,2,19-23}$ As such, close clinical follow up is particularly relevant during the 
12-week window after vector infusion. Lastly, although the AAV8FIX trial, and subsequently, others, reported the ability to control an immune response and maintain at least partial transgene expression with oral steroids, use of immunomodulation has not always

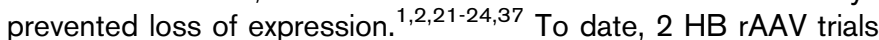
sponsored by Shire ${ }^{20}$ and The Children's Hospital of Philadelphia (Katherine A. High, unpublished data) lost transgene expression, despite immunomodulatory intervention showing that steroids are not universally effective.

However, after responsiveness to steroids is established for a given vector, the need for a short course of steroids is not necessarily a shortcoming but highlights the need to rapidly and accurately recognize a capsid immune response. There is no currently available clinical laboratory test to directly diagnose an AAV capsid immune response. Prior and currently used measures to identify a deleterious capsid immune response include liver function studies, FIX:C or factor VIII activity (FVIII:C; a measure of transgene expression), and interferon- $\gamma$ ELISPOT assays. The interferon- $\gamma$ ELISPOT testing is an in vitro assay, in which participant peripheral blood mononuclear cells are isolated and exposed to capsid peptide pools; interferon- $\gamma$ release is then quantitated. ${ }^{35}$ The testing is intended as an indirect measure of CD8 + T-cell activation to capsid peptides and is, at this point, a research test that is not standardized across ongoing clinical trials. Additionally, results are not available for $\sim 4$ days after sample collection and may not be available at the time of necessary clinical decision making around use of steroids. Lastly, in vitro results and in vivo physiology do not always correlate. Thus, ELISPOT results should be interpreted in conjunction with the factor level and liver function tests (LFTs). The combination of a declining factor level, rise in LFTs, and positive interferon- $\gamma$ ELISPOT results are concerning for evidence of a capsid immune response.

\section{Vector design and delivery}

In addition to the use of steroids in the setting of a capsid immune response, the AAV8-FIX trial integrated changes to the vector design and delivery that have been incorporated into subsequent trials, including peripheral intravenous infusion that is now the universal route of administration in current trials (Figure 2). In keeping with discovery of alternative AAV serotypes, the group used an AAV8 vector with liver-specific tropism. ${ }^{15}$ The group also incorporated the use of a self-complementary vector genome. Selfcomplementary adeno-associated virus (scAAV) vector genomes have a covalently closed hairpin that connects 2 monomer singlestranded genomes in an inverted or head-to-head dimer. The theoretical advantage of using scAAV is that the 2 genomes would reanneal on target cell transduction, leading to rapid and enhanced gene expression without the need for second strand synthesis, ${ }^{38}$ which was supported in preclinical investigation. ${ }^{39}$ However, subsequent work showed vulnerabilities to the accuracy of the method used to titer scAAV, such that underestimation of the vector titer may have contributed to preclinical observations. ${ }^{40}$ The role of scAAV vs a single-stranded adeno-associated virus (ssAAV) cassette in transduction efficiency is not clear and is only applicable to FIX gene transfer, because the complementary DNA of BDD-FVIII does not fit into an scAAV vector design. All current FIX trials use an scAAV vector, except that in investigation in a trial sponsored by Spark Therapeutics, which uses a single-stranded rAAV vector (ssAAV). The FIX protein antigen expression of the Spark

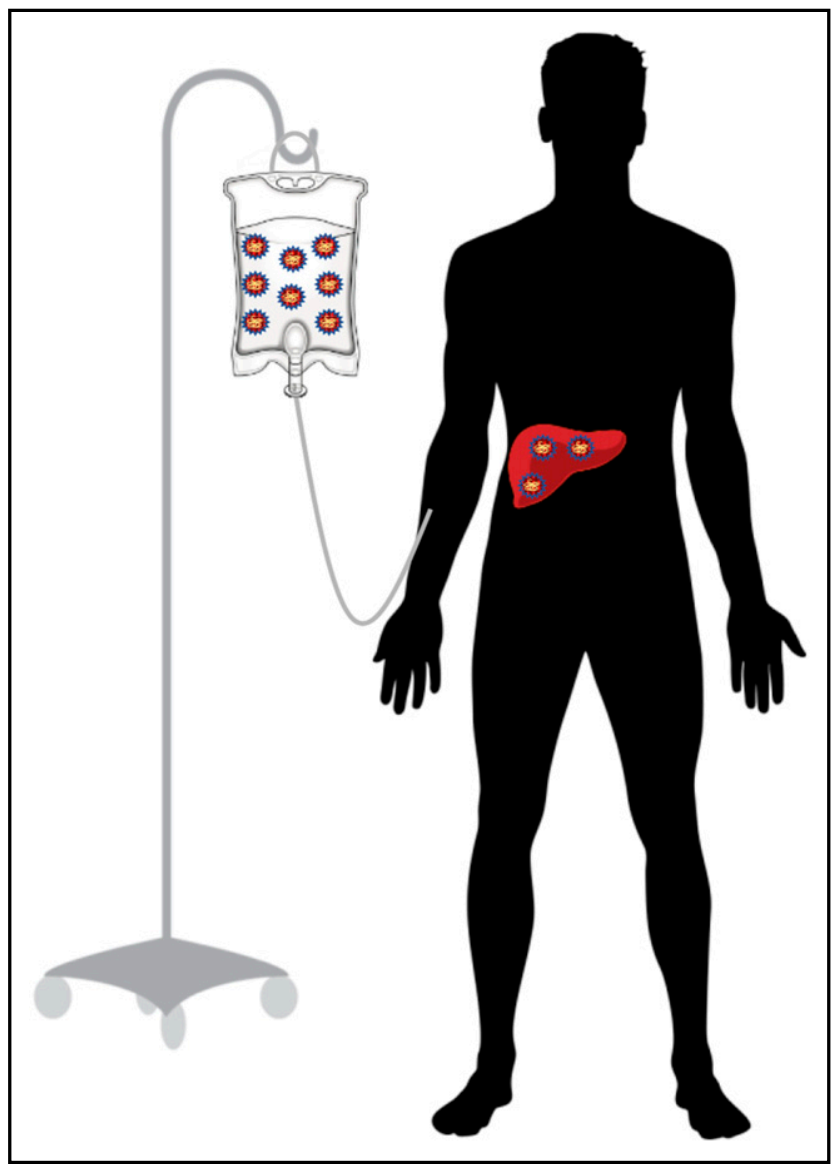

Figure 2. Peripheral administration of rAAV vector for liver-directed gene transfer for $H A$ or $H B$.

ssAAV-FIX vector at a dose of $5 \times 10^{11} \mathrm{vg} / \mathrm{kg}^{21,22}$ approximates that observed in a fourfold higher dose $\left(2 \times 10^{12} \mathrm{vg} / \mathrm{kg}\right)$ of the AAV8-FIX trial, ${ }^{1,2}$ suggesting that there may not be an advantage to using an scAAV vector. However, given other differences between the 2 vectors, no definitive conclusion can be drawn.

\section{Current trials}

The potential for gene transfer to revolutionize hemophilia care is evidenced by the number of ongoing early-phase clinical trials (Tables 1 and 2). Indeed, groups have now reported factor activity levels into the normal range after both $\mathrm{FVIII}^{24}$ and $\mathrm{FIX}^{21,22}$ gene transfer. Importantly, however, these results have only been presented in abstracts, or updated data have been made available by company press release, limiting published reports to the AAV2FIX and AAV8-FIX trials. ${ }^{1,2,19}$ Appropriate caution is warranted in attempting to draw conclusions from unpublished or interim results.

All current trials are phase $1 / 2$ studies with similar inclusion and exclusion criteria. These inclusion/exclusion criteria outline that trial participants must be adult males ( $\geq 18$ years old) with moderate or severe hemophilia; negative or low-titer AAV NAb; no evidence of active hepatitis $B$ virus or hepatitis $C$ virus (HCV) infection; an adequate CD4 + count if HIV-positive; and a minimum number of factor exposure days (typically $>50$ ) with no current or prior inhibitor. The greatest safety concern of 


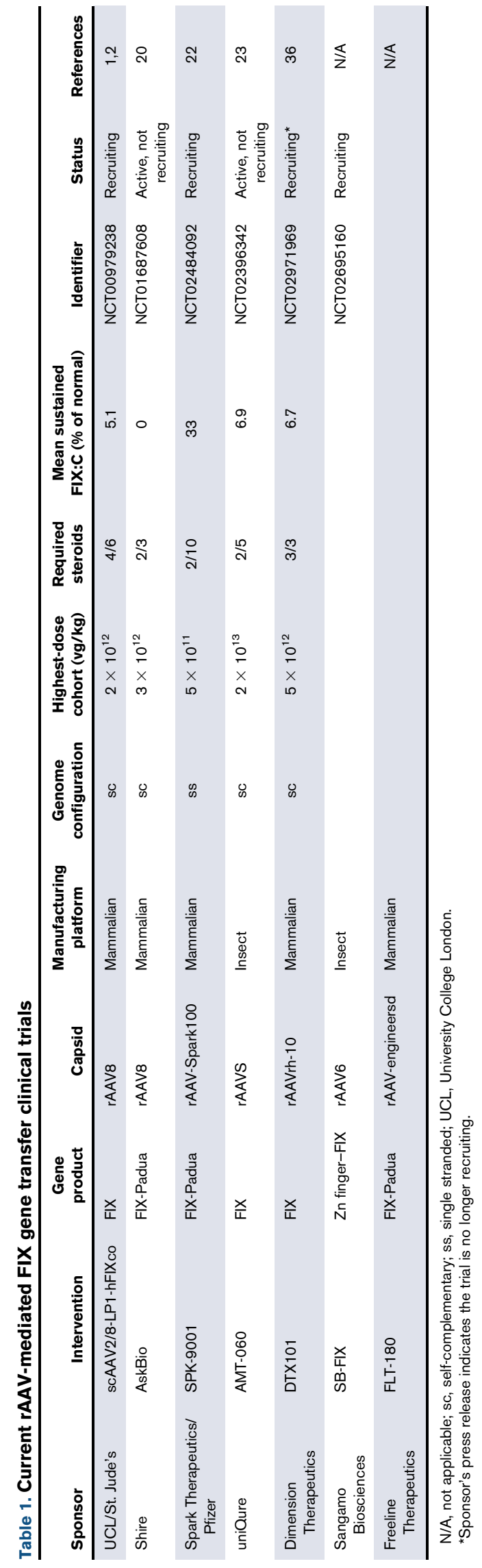

liver-directed gene transfer is liver toxicity. The safety of liverdirected rAAV-mediated gene transfer in patients with underlying severe liver disease is not known. This is particularly relevant, because recent Centers for Disease Control and Prevention data show $>80 \%$ of hemophilia patients $\geq 35$ years old and $>90 \%$ of those $\geq 40$ years old contracted HCV. ${ }^{41}$ The recent incorporation of ledipasvir/sofosbuvir, which nearly universally clears $\mathrm{HCV}$, into widespread clinical practice and demonstrated regression of liver fibrosis after HCV clearance mean that a broader hemophilia patient population is eligible for gene transfer trials. ${ }^{42,43}$ This said, the degree of liver fibrosis as it relates to both short- and long-term safety and efficacy of liver-directed rAAV gene transfer is not clear and remains an important question.

The most common reason for clinical trial exclusion is the presence of preexisting AAV NAb. After vector infusion, subjects develop high-titer NAb, thereby precluding the ability to undergo repeat vector infusion. Strategies to overcome preexisting AAV NAbs or prevent their development have been previously reviewed. ${ }^{44}$ However, at the current state of scientific development, intravenous rAAV vector infusion is only available as a one-time infusion. Given the pace of progress in rAAV hemophilia gene therapy, it is my practice to discuss the development of AAV NAb after vector infusion as a risk of trial enrollment during informed consent. The development of AAV NAb after vector infusion may be particularly important when considering extending this therapy to pediatric patients who may require repeat infusion to accommodate hepatic growth (hepatocyte division) and expanded plasma volume. Lastly, given the ability of NAb to block transduction, a sensitive AAV NAb assay is particularly important for predicting transgene transduction, expression, and therefore, therapeutic efficacy. As previously discussed, differences in baseline AAV NAb titers would be expected to result in variable transgene expression within a dose cohort.

Although inclusion/exclusion criteria are relatively similar among trials, the rAAV vector may differ considerably for reasons that are both disclosed (outlined in Tables 1 and 2) and not disclosed, such as differences in genome configuration and vector manufacturing previously mentioned. Given that all trials are phase $1 / 2$, they aim to evaluate safety, which is primarily focused on evidence of liver toxicity. Acknowledging the known strong correlation of factor activity and expected phenotype, preliminary efficacy end points have predictable findings based on transgene expression and activity and include reduction in bleeding and factor use. No current trials have a control group, limiting conclusions that can be drawn from preliminary efficacy data; however, most trials have incorporated retrospective bleeding and factor logs to allow for pre- and postvector clinical data comparison. Nonetheless, although current trials aim to evaluate safety, the success of several early-phase trials along with current realization that, at this time, enrollment in rAAV gene transfer trials is a one-time choice, raise the question of what is the minimally desired factor level. Natural history data indicate that patients with endogenous FVIII:C $\geq 12 \%$ of normal do not experience spontaneous bleeding. ${ }^{45}$ Although these data cannot be universally extrapolated for all patients, particularly those with severe arthropathy, and may not necessarily approximate similar requirements to eliminate spontaneous bleeding in $\mathrm{HB}$, this may at least provide a rationale for the minimally desired targeted transgene expression. 


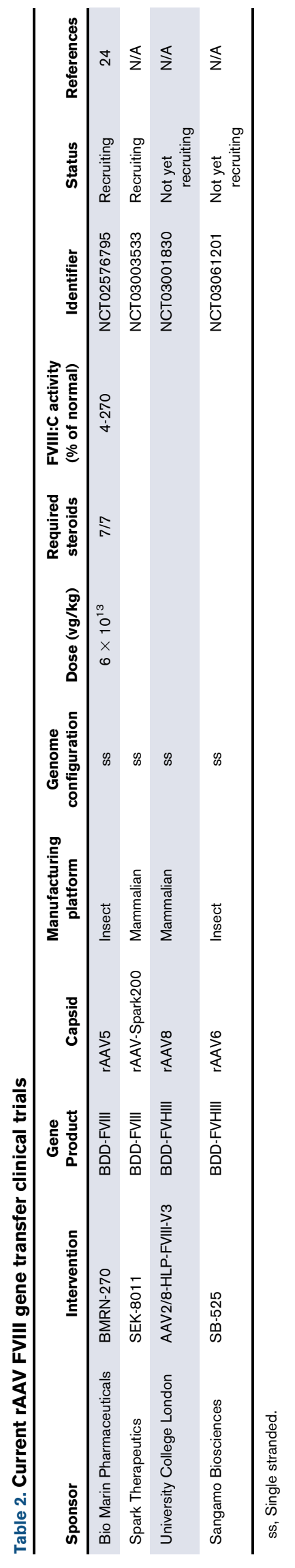

\section{Current rAAV-FIX trials}

After the first reported success of sustained FIX expression in HB patients, there are now 4 additional ongoing clinical trials in $\mathrm{HB}$ (Table 1). Among the next generation trials, 2 of 4 incorporated use of an FIX-Padua transgene. FIX-Padua (FIX-R338L) is a naturally occurring missense mutation in the FIX catalytic domain, resulting in approximately eightfold greater specific activity. ${ }^{46}$ Preliminary biochemical characterization reported that the greater specific activity of FIX-R338L was caused by enhanced function in the intrinsic Xase with similar FIX activation and inactivation as wild-type FIX, thereby supporting the safety of FIX-R338L as a therapeutic. ${ }^{47}$ Although use of a variant transgene raises a theoretical concern for possible inhibitor development, preclinical studies showed that FIX-R338L expression after liver-directed rAAV-mediated gene transfer did not result in inhibitor formation and furthermore, was able to induce tolerance in $\mathrm{HB}$ canine models with preexisting inhibitors. ${ }^{48,49}$ Thus far, 18 total HB subjects have been reported to express FIX-R338L after gene transfer, including 11 with sustained expression, and no subjects developed inhibitors to either wild-type WT-FIX or FIXR338L. ${ }^{20-22}$ In addition to the transgene, disclosed differences between the vectors include the capsid, genome configuration (ssAAV or scAAV cassette), and manufacturing platform. Lastly, among the trials, there is a 40 -fold difference between the vector dose of the therapeutic and highest-dose cohorts reported $\left(5 \times 10^{11}\right.$ to $\left.2 \times 10^{13} \mathrm{vg} / \mathrm{kg}\right)$.

Recognizing that the risk of an anti-AAV capsid immune response correlates with vector dose, one way to minimize the risk of an immune response would be to use the lowest vector dose possible. This strategy was used by Spark Therapeutics with their vector, SPK-9001, which consists of a bioengineered capsid (AAV-Spark100) that has a biodistribution profile similar to AAV, ${ }^{50}$ human $\alpha$-1-antitrypsin promotor, and single-stranded DNA configuration. ${ }^{21,22}$ Preliminary results of 10 patients with 16 to 52 weeks of follow up showed sustained average transgene-derived FIX:C of $\sim 30 \%$ of normal with a vector dose of $5 \times 10^{11} \mathrm{vg} / \mathrm{kg}$. These results represent the highest levels of sustained FIX expression after gene transfer at the lowest vector dose. Among the 10 patients, 2 required a course of oral steroids, representing the lowest frequency of immune response of all AAV hemophilia trials to date. These data support the strategy of using the lowest possible dose to minimize risk of a dose-dependent capsid immune response. Preliminary efficacy data showed a significant reduction in annualized bleeding rate and $99 \%$ reduction in factor use. ${ }^{22}$ Plans for phase 3 trial investigation of the SPK-9001 vector are in process.

The remaining $\mathrm{HB}$ gene therapy trials used higher vector doses, achieved lower levels of transgene-derived FIX:C, and had a greater frequency of steroid intervention. These 3 trials have described steroid intervention as not necessarily indicative of an rAAV capsid immune response. Although prior data support a decline in FIX:C and transaminase elevation as consistent with a capsid immune response, it is possible that new data from these trials support alternative models that have not yet been reported. $^{1,2,19,35}$ Before the initiation of the Spark clinical trial, Baxalta/Shire investigated an scAAV8 FIX-R338L vector, in which transgene expression was lost in all but one subject in the middose cohort $\left(1 \times 10^{12} \mathrm{vg} / \mathrm{kg}\right)$, despite use of oral steroid 
intervention in the highest-dose cohort. ${ }^{20}$ Although the trial is no longer recruiting, Monahan et $\mathrm{al}^{20}$ importantly established that there was no evidence of inhibitor formation after FIX-R338L expression.

The remaining 3 trials use an scAAV vector expressing wildtype FIX. Results from the trial sponsored by St. Jude's Research Hospital have been previously discussed and published. ${ }^{1,2}$ UniQure is developing an scAAV5-FIX vector and has presented data on 10 patients from 2 dose cohorts. Mean reported transgene-derived FIX:C of the highest-dose cohort $\left(2 \times 10^{13} \mathrm{vg} / \mathrm{kg}\right)$ is $6.9 \%$ of normal, and 2 of 5 patients were treated with oral steroids without loss of FIX:C. In the high-dose cohort, all 4 patients previously maintained on prophylaxis were able to discontinue recombinant factor replacement. ${ }^{23}$ Information on the remaining HB trial sponsored by Dimension Therapeutics is only available by press release. The company developed an scAAV-FIX vector consisting of a bioengineered capsid AAVrh-10 and dose escalated to a vector dose of $5 \times 10^{12} \mathrm{vg} / \mathrm{kg} ; 3$ of 3 subjects at the highest-dose cohort required oral steroids, wherein some, but not all, transgene expression was rescued, resulting in sustained reported transgene-derived FIX:C of $5 \%$ to $8 \%$. The company has since decided to discontinue additional development of the vector. ${ }^{37}$ The remaining open HB AAV gene transfer trial is sponsored by Sangamo Biosciences and is a genome editing approach involving coadministration of AAV vectors to deliver a zinc finger nuclease and a donor FIX transgene. Although preclinical data are published, clinical trial data are not available. ${ }^{51}$ An additional FIX AAV-mediated gene transfer trial has been proposed by Freeline Therapeutics but is not yet open.

\section{Current rAAV-FVIII trials}

Biological differences between the FIX and FVIII proteins present specific challenges to HA gene transfer. First, to meet ssAAV packaging constraints, the large nonfunctional $B$ domain (accounting for $40 \%$ of the protein) can be truncated to an SQ linker consisting of 14 aa (BDD-FVIII). Recombinant BDD-FVIII maintains full cofactor function, results in an $\sim 30 \%$ increase in secreted protein from the synthesizing cell, and has been in clinical use for approximately 2 decades with no observed increased incidence in inhibitor development. ${ }^{52,53}$ Second, for unclear reasons, FVIII is poorly expressed and inefficiently secreted. Thus, although the physiologic plasma concentration of FVIII $(1 \mathrm{nM})$ is much lower than that of FIX (90 nM), inefficient FVIII expression has, until recently, posed the greatest challenge to advancing FVIII gene transfer. ${ }^{54}$ Third, the FVIII protein seems to be inherently immunogenic, with an incidence of inhibitor development of $20 \%$ to $30 \%$ in severe patients vs $\sim 10 \%$ in severe $\mathrm{HB}$, which raises concern for possible inhibitor development after gene transfer ${ }^{55,56}$ Notably, however, like FIX-Padua, liver-directed rAAV-mediated FVIII gene transfer has shown ability to induce tolerance in HA canine models with preexisting inhibitors. ${ }^{57}$ Thus, preclinical evidence supports that liver-directed gene transfer conferring continuous uninterrupted expression of FVIII induces tolerance. Extending gene transfer to HA patients with inhibitors is particularly exciting, because they currently experience the greatest morbidity and mortality among hemophilia patients and because most benefit from a novel therapeutic. $^{58}$
Currently, there are 2 active FVIII gene transfer trials. Preliminary data from the trial sponsored by Biomarin were initially presented at the July 2016 World Federation of Hemophilia meeting outlining use of an AAV5 BDD-FVIII vector. Preliminary transgene expression data among the three dose cohorts included no detectable expression in the single subject infused at $6 \times 10^{12} \mathrm{vg} / \mathrm{kg}$; FVIII:C $>2 \%$ of normal in the single subject infused at $2 \times 10^{13}$ $\mathrm{vg} / \mathrm{kg}$; and FVIII:C between $4 \%$ and $270 \%$ of normal among 7 subjects infused at $6 \times 10^{13} \mathrm{vg} / \mathrm{kg}$. All subjects in the highest-dose cohort were treated with oral steroids. ${ }^{24}$ In January 2017, the group presented updated data of the high-dose cohort subjects at the J. P. Morgan Healthcare Conference and in a concurrent press release. Encompassing a follow-up interval of 34 to 50 weeks, subjects maintained transgene expression off oral steroids with FVIII:C that ranged from $16 \%$ to $222 \%$ of normal, wherein FVIII:C decreased in three subjects since the original data presentation in July 2016. Preliminary efficacy data reported included a $91 \%$ decline in annual bleed rate and 98\% decrease in prophylactic infusions. Prior observations and published studies with AAV-FIX have shown a more linear vector dose response and less variability in transgene expression within dose cohorts. ${ }^{1,2,19,21,22}$ The mechanisms for the disparate levels of FVIII:C at the same vector dose and lack of a vector dose response have not been communicated. Although this potentially curative vector is quite promising, several questions remain in understanding this complex data and how to address supratherapeutic transgene expression. In addition to the Biomarin trial, Spark Therapeutics opened a FVIII trial in January 2017, and several additional rAAV FVIII trials are anticipated to open by the end of 2017 (Table 2).

\section{Conclusion}

Recent replicable successful clinical trial results support that gene therapy may soon deliver on its promise to alter the paradigm of hemophilia care. Although some of the variation in clinical trial observation is predictable based on differences in the transgene and vector dose, full understanding of discordant results is not clear. Insight needed to fully understand the etiology of variable trial results may be in appreciating differences in the sensitivity of individual trial immunomonitoring assay monitoring (NAb and ELISPOT assays) and the more finite contribution of particular vectors properties. Despite variability in results, recent clinical trial advances leave us with the optimism to consider the next steps of how this therapy may be incorporated into hemophilia care. Specifically, what will be the minimum bar for transgene-derived factor activity, and how can we extend this therapy to more patients? Where gene therapy may ultimately sit within the growing therapeutic repertoire for hemophilia may be largely dependent on expanding the patient population for whom gene transfer is available. This will require overcoming current obstacles, including (1) an ability to treat patients with preexisting AAV NAb; (2) establishing therapeutic durability in pediatric patients, who may require ability to reinfuse vector; (3) establishing the safety of liverdirected gene transfer in the setting of underlying liver disease; and (4) extending therapy to inhibitor patients for which preclinical data support liver-directed gene transfer to induce tolerance. Lastly, although there are an estimated 400000 people worldwide with hemophilia, $\sim 75 \%$ do not have access to routine care. ${ }^{59}$ A one-time treatment to ameliorate disease phenotype is particularly attractive for this patient population. Although challenges remain, the success 
of ongoing clinical trial work supports that rAAV-based gene transfer for hemophilia will advance into future clinical care.

\section{Acknowledgments}

L.A.G. thanks Benjamin Samelson-Jones and Katherine High for their thoughtful review of the manuscript.

L.A.G. is the recipient of young investigator awards from the National Blood Foundation and the Hemostasis and Thrombosis Research Society.

\section{Authorship}

Contribution: L.A.G. wrote the paper and prepared the figures and tables.

Conflict-of-interest disclosure: L.A.G. has consulted for Pfizer. Off-label drug use: None disclosed.

Correspondence: Lindsey A. George, Division of Hematology, The Children's Hospital of Philadelphia, 3501 Civic Center Blvd, Colket Translational Research Bldg, Room 5016, Philadelphia, PA 19104-4313; e-mail: georgel@email.chop.edu.

\section{References}

1. Nathwani AC, Tuddenham EG, Rangarajan S, et al. Adenovirus-associated virus vector-mediated gene transfer in hemophilia B. N Engl J Med. 2011; 365(25):2357-2365.

2. Nathwani AC, Reiss UM, Tuddenham EG, et al. Long-term safety and efficacy of factor IX gene therapy in hemophilia B. N Engl J Med. 2014;371(21):1994-2004.

3. Mannucci PM, Tuddenham EG. The hemophilias-from royal genes to gene therapy. N Engl J Med. 2001;344(23):1773-1779.

4. Mingozzi F, High KA. Therapeutic in vivo gene transfer for genetic disease using AAV: progress and challenges. Nat Rev Genet. 2011;12(5):341-355.

5. Ohmori T, Mizukami H, Ozawa K, Sakata Y, Nishimura S. New approaches to gene and cell therapy for hemophilia. J Thromb Haemost. 2015;13(suppl 1):S133-S142.

6. Kattenhorn LM, Tipper CH, Stoica L, et al. Adeno-associated virus gene therapy for liver disease. Hum Gene Ther. 2016;27(12):947-961.

7. Sarkar R, Tetreault R, Gao G, et al. Total correction of hemophilia A mice with canine FVIII using an AAV 8 serotype. Blood. 2004;103(4):1253-1260.

8. Mount JD, Herzog RW, Tillson DM, et al. Sustained phenotypic correction of hemophilia B dogs with a factor IX null mutation by liver-directed gene therapy. Blood. 2002;99(8):2670-2676.

9. Sabatino DE, Lange AM, Altynova ES, et al. Efficacy and safety of long-term prophylaxis in severe hemophilia A dogs following liver gene therapy using AAV vectors. Mol Ther. 2011;19(3):442-449.

10. Dong JY, Fan PD, Frizzell RA. Quantitative analysis of the packaging capacity of recombinant adeno-associated virus. Hum Gene Ther. 1996;7(17):2101-2112.

11. Wright JF. Transient transfection methods for clinical adeno-associated viral vector production. Hum Gene Ther. 2009;20(7):698-706.

12. Negrete A, Yang LC, Mendez AF, Levy JR, Kotin RM. Economized large-scale production of high yield of rAAV for gene therapy applications exploiting baculovirus expression system. J Gene Med. 2007;9(11):938-948.

13. Paulk NK, Rumachik NG, Adams CM, et al. rAAV is extensively and differentially post-translationally modified in human versus insect cell line production methods. Mol Ther. 2017;25(5 supp1):46.

14. Faust SM, Bell P, Cutler BJ, et al. CpG-depleted adeno-associated virus vectors evade immune detection. J Clin Invest. 2013;123(7):2994-3001.

15. Gao GP, Alvira MR, Wang L, Calcedo R, Johnston J, Wilson JM. Novel adeno-associated viruses from rhesus monkeys as vectors for human gene therapy. Proc Natl Acad Sci USA. 2002;99(18):11854-11859.

16. European Medicines Agency. Alipogene tiparvovec. http://www.ema.europa.eu/docs/en_GB/document_library/EPAR_-_Public_assessment_report/ human/002145/WC500135476.pdf. Accessed 19 May 2017.

17. European Medicines Agency. Strimvelis. http://www.ema.europa.eu/docs/en_GB/document_library/EPAR_-_Public_assessment_report/human/ 003854/WC500208201.pdf. Accessed 18 May 2017.

18. Daya S, Berns KI. Gene therapy using adeno-associated virus vectors. Clin Microbiol Rev. 2008;21(4):583-593.

19. Manno CS, Pierce GF, Arruda VR, et al. Successful transduction of liver in hemophilia by AAV-Factor IX and limitations imposed by the host immune response [published correction appears in Nat Med. 2006;12(5):592]. Nat Med. 2006;12(3):342-347.

20. Monahan PE, Walsh CE, Powell JS, et al. Update on phase $1 / 2$ open-label trial of BAX335, an adeno-associated virus 8 (AAV8) vector-based gene therapy for program for phemophilia B. J Thromb Haemost. 2015;13(suppl 2):87.

21. George LA, Sullivan SK, Giermasz A, et al. Spk-9001: adeno-associated virus mediated gene transfer for hemophilia B achieves sustained mean factor IX activity levels of $>30 \%$ without immunosuppression. Blood. 2016;128(22):3.

22. George LA, Sullivan SK, Giermasz A, et al. SPK-9001: adeno-associated virus mediated gene transfer for hemophilia B at the low vector dose achieved sustained, continuous factor IX activity levels adequate for endogenous prophylaxis preventing bleeding episodes and reducing the risk of immune response. Mol Ther. 2017;25(5 supp1):331.

23. Leebeek FW, Tangelder M, Meijer K, et al. Interim results from a dose escalating study of AMT-060 (AAV5-hFIX) gene transfer in adult patients with severe hemophilia B. Blood. 2016;128(22):2314.

24. Pasi J, Wong W, Rangarajan S. Interim results of an open-label, phase 1/2 study of BMN 270, an AAV5-FVIII gene transfer in severe hemophilia A. Haemophilia. 2016;22(suppl 4):151-152.

25. Rutledge EA, Russell DW. Adeno-associated virus vector integration junctions. J Virol. 1997;71(11):8429-8436.

26. Nakai H, Iwaki Y, Kay MA, Couto LB. Isolation of recombinant adeno-associated virus vector-cellular DNA junctions from mouse liver. J Virol. 1999;73(7):5438-5447. 
27. Donsante A, Miller DG, Li Y, et al. AAV vector integration sites in mouse hepatocellular carcinoma. Science. 2007;317(5837):477.

28. Chandler RJ, LaFave MC, Varshney GK, et al. Vector design influences hepatic genotoxicity after adeno-associated virus gene therapy. $J$ Clin Invest. 2015;125(2):870-880.

29. Nault JC, Datta S, Imbeaud S, et al. Recurrent AAV2-related insertional mutagenesis in human hepatocellular carcinomas. Nat Genet. 2015;47(10):1187-1193.

30. Weitzman MD, Linden RM. Adeno-associated virus biology. Methods Mol Biol. 2011;807:1-23.

31. Nichols TC, Whitford MH, Arruda VR, Stedman HH, Kay MA, High KA. Translational data from adeno-associated virus-mediated gene therapy of hemophilia B in dogs. Hum Gene Ther Clin Dev. 2015;26(1):5-14.

32. Wang L, Zoppè M, Hackeng TM, Griffin JH, Lee KF, Verma IM. A factor IX-deficient mouse model for hemophilia B gene therapy. Proc Nat/ Acad Sci USA. 1997;94(21):11563-11566.

33. Manno CS, Chew AJ, Hutchison S, et al. AAV-mediated factor IX gene transfer to skeletal muscle in patients with severe hemophilia B. Blood. 2003; 101(8):2963-2972.

34. Calcedo R, Vandenberghe LH, Gao G, Lin J, Wilson JM. Worldwide epidemiology of neutralizing antibodies to adeno-associated viruses. J Infect Dis. 2009;199(3):381-390.

35. Mingozzi F, Maus MV, Hui DJ, et al. CD8(+) T-cell responses to adeno-associated virus capsid in humans. Nat Med. 2007;13(4):419-422.

36. Rogers GL, Shirley JL, Zolotukhin I, et al. Plasmacytoid and conventional dendritic cells cooperate in crosspriming AAV capsid-specific CD8(+) T cells. Blood. 2017;129(24):3184-3195.

37. http://investors.dimensiontx.com/phoenix.zhtml?c=254192\&p=irol-newsArticle\&ID=2271810. Accessed 9 May 2017.

38. McCarty DM, Fu H, Monahan PE, Toulson CE, Naik P, Samulski RJ. Adeno-associated virus terminal repeat (TR) mutant generates self-complementary vectors to overcome the rate-limiting step to transduction in vivo. Gene Ther. 2003;10(26):2112-2118.

39. Nathwani AC, Gray JT, $\mathrm{Ng} \mathrm{CY,} \mathrm{et} \mathrm{al.} \mathrm{Self-complementary} \mathrm{adeno-associated} \mathrm{virus} \mathrm{vectors} \mathrm{containing} \mathrm{a} \mathrm{novel} \mathrm{liver-specific} \mathrm{human} \mathrm{factor} \mathrm{IX} \mathrm{expression}$ cassette enable highly efficient transduction of murine and nonhuman primate liver. Blood. 2006;107(7):2653-2661.

40. Fagone P, Wright JF, Nathwani AC, Nienhuis AW, Davidoff AM, Gray JT. Systemic errors in quantitative polymerase chain reaction titration of selfcomplementary adeno-associated viral vectors and improved alternative methods. Hum Gene Ther Methods. 2012;23(1):1-7.

41. Mazepa MA, Monahan PE, Baker JR, Riske BK, Soucie JM; US Hemophilia Treatment Center Network. Men with severe hemophilia in the United States: birth cohort analysis of a large national database. Blood. 2016;127(24):3073-3081.

42. Kowdley KV, Gordon SC, Reddy KR, et al; ION-3 Investigators. Ledipasvir and sofosbuvir for 8 or 12 weeks for chronic HCV without cirrhosis. N Engl J Med. 2014;370(20):1879-1888.

43. Chekuri S, Nickerson J, Bichoupan K, et al. Liver stiffness decreases rapidly in response to successful hepatitis C treatment and then plateaus. PLoS One. 2016;11(7):e0159413.

44. Mingozzi F, High KA. Immune responses to AAV vectors: overcoming barriers to successful gene therapy. Blood. 2013;122(1):23-36.

45. Den Uijl IE, Mauser Bunschoten EP, Roosendaal G, et al. Clinical severity of haemophilia A: does the classification of the 1950s still stand? Haemophilia. 2011;17(6):849-853.

46. Simioni P, Tormene D, Tognin G, et al. X-linked thrombophilia with a mutant factor IX (factor IX Padua). N Engl J Med. 2009;361(17):1671-1675.

47. Samelson-Jones BJ, Finn JD, Camire RM, Arruda VR. New hyperactive factor IX variants bioengineered based upon biochemical studies of factor IX Padua. Blood. 2016;128(22):1384.

48. Molino M, Di Lallo M, de Gaetano G, Cerletti C. Intracellular Ca2 + rise in human platelets induced by polymorphonuclear-leucocyte-derived cathepsin G. Biochem J. 1992;288(pt 3):741-745.

49. Crudele JM, Finn JD, Siner Jl, et al. AAV liver expression of FIX-Padua prevents and eradicates FIX inhibitor without increasing thrombogenicity in hemophilia B dogs and mice. Blood. 2015;125(10):1553-1561.

50. Anguela XM, Toso R, Cuoto LB, et al. Safety and efficacy of a novel AAV vector for treatment of hemophilia B. J Thromb Haemost. 2015;13(suppl 2):324-325.

51. Li H, Haurigot V, Doyon Y, et al. In vivo genome editing restores haemostasis in a mouse model of haemophilia. Nature. 2011;475(7355):217-221.

52. Pittman DD, Alderman EM, Tomkinson KN, Wang JH, Giles AR, Kaufman RJ. Biochemical, immunological, and in vivo functional characterization of B-domain-deleted factor VIII. Blood. 1993;81(11):2925-2935.

53. Gringeri A, Tagliaferri A, Tagariello G, Morfini M, Santagostino E, Mannucci P; ReFacto-AICE Study Group. Efficacy and inhibitor development in previously treated patients with haemophilia A switched to a B domain-deleted recombinant factor VIII. Br J Haematol. 2004;126(3):398-404.

54. Marder VJ, Aird WC, Bennett JS, Schulman S, White GC. Hemostasis and Thrombosis: Basic Principles and Clinical Practice. 6th ed. Philadelphia, PA: Lippincott Williams \& Wilkins; 2013.

55. Eckhardt CL, van Velzen AS, Peters M, et al; INSIGHT Study Group. Factor VIII gene (F8) mutation and risk of inhibitor development in nonsevere hemophilia A. Blood. 2013;122(11):1954-1962.

56. DiMichele D. Inhibitor development in haemophilia B: an orphan disease in need of attention. Br J Haematol. 2007;138(3):305-315.

57. Finn JD, Ozelo MC, Sabatino DE, et al. Eradication of neutralizing antibodies to factor VIII in canine hemophilia A after liver gene therapy. Blood. 2010; 116(26):5842-5848.

58. Walsh CE, Soucie JM, Miller CH; United States Hemophilia Treatment Center Network. Impact of inhibitors on hemophilia A mortality in the United States. Am J Hematol. 2015;90(5):400-405.

59. Skinner MW. Gene therapy for hemophilia: addressing the coming challenges of affordability and accessibility. Mo/ Ther. 2013;21(1):1-2. 